Check for updates

Cite this: RSC Adv., 2019, 9, 9633

\title{
Novel graphene-like two-dimensional bilayer germanene dioxide: electronic structure and optical properties
}

\author{
Yan-Mei Dou, Chang-Wen Zhang, Ping Li and Pei-Ji Wang (D) *
}

Using ab initio calculations, we present a two-dimensional (2D) $\alpha$-2D-germanene dioxide material with an ideal $\mathrm{sp}^{3}$ bonding network which possesses a large band gap up to $2.50 \mathrm{eV}$. The phonon dispersion curves and molecular dynamics (MD) simulation under the chosen parameters suggest that the novel 2D structure is stable. The dielectric function and absorption spectrum also show the consistent band gap within the electronic structure diagram, suggesting possible application as an ultraviolet light optical detector. The calculated carrier mobility of $4.09 \times 10^{3} \mathrm{~cm}^{2} \mathrm{~V}^{-1} \mathrm{~s}^{-1}$ can be observed along the $x$ direction, which is much higher than that of $\mathrm{MoS}_{2}\left(\sim 3.0 \mathrm{~cm}^{2} \mathrm{~V}^{-1} \mathrm{~s}^{-1}\right)$. Finally, we found that $\alpha$-2D-germanene dioxide could potentially act as an ideal monolayer insulator in so-called van der Waals (vdW) heterostructure devices. These findings expand the potential applications of the emerging field of 2D $\alpha$-2D-germanene dioxide materials in nanoelectronics.

Received 18th January 2019

Accepted 11th March 2019

DOI: $10.1039 / c 9 r a 00450 e$

rsc.li/rsc-advances

\section{Introduction}

Silicon dioxide $\left(\mathrm{SiO}_{2}\right)$ is one of the most abundant materials on Earth. ${ }^{1} \mathrm{SiO}_{2}$, normally existing in bulk crystalline and amorphous forms such as quartz (including $\alpha$ - and $\beta$-quartz), cristobalite ( $\alpha$ - and $\beta$-cristobalite) and glassy silica, was recently found to possess a two-dimensional (2D) structure. ${ }^{1-3}$ In general, in all known silica compounds, the silicon has a fourfold tetrahedron configuration with $\mathrm{sp}^{3}$ hybridization. The miscellaneous phases of silica mainly originate from the spacestacking form of the Si-O tetrahedron. ${ }^{4-7}$ In recent findings, the thermal, dynamic and mechanical stabilities of some 2D silica structures are compared with typical bulk silica. ${ }^{8,9}$ Silica films can act as dielectric layers in integrated circuits and supporting substrates for catalysis because they spontaneously form on a clean silicon surface when the surface is exposed to air.

Recently, Löffler et al. have experimentally grown and determined the structure of crystalline silica sheets on $\mathrm{Ru}$ (0001); namely a silica bilayer has recently been synthesized..$^{10-15}$ Furthermore, this hexagonal quasi-2D silica can even be supported by graphene. ${ }^{16}$ In other 2D materials, including $\mathrm{MoS}_{2}$ and $\mathrm{WS}_{2}$, h-BN, silicene, germanene, MXene and phosphorene, electrons have freedom only in the 2D plane because of the quantum confinement effect, which could give rise to new phenomena in physics. ${ }^{17,18}$ Germanene dioxide has the same electronic form as silicon dioxide; therefore, as the study matured, we focused on germanene from the same main group.

School of Physics and Technology, University of Jinan, Jinan, Shandong, 250022, People's Republic of China. E-mail: ss_wangpj@ujn.edu.cn
Other 2D materials also have a high carrier mobility of up to $10^{5} \mathrm{~cm}^{2} \mathrm{~V}^{-1} \mathrm{~s}^{-1}$ (ref. 19 and 20) and novel in-plane negative Poisson's ratio, ${ }^{21}$ while the excellent bandgap is also beneficial to applications in optoelectronics. ${ }^{22}$ The germanene monolayer has been found to be an insulator with a large bandgap, as well as high carrier mobility (of the order of $10^{5} \mathrm{~cm}^{2} \mathrm{~V}^{-1} \mathrm{~s}^{-1}$ ) due to its linear band dispersion near the Fermi level $\left(E_{\mathrm{F}}\right)$ at the $K$ point. It has a low-buckled $(0.84 \AA)$ structure compared with silicene due to the weak p-p interactions and distinct coupling of $\mathrm{s}$ and $\mathrm{p}$ bonds between Ge atoms, ${ }^{23-26}$ giving rise to new characteristics beyond silica, such as detectable quantum spin Hall (QSH) and valley-polarized quantum anomalous Hall (QAH) states, for example..$^{27,28}$ In addition, Gao et al. ${ }^{29}$ showed that it can be used for infrared materials, precision instruments and catalysts. Several remarkable features of germanene have been reported. ${ }^{26,30,31}$

Despite the extensive efforts of 2D germanene dioxide monolayers, ${ }^{32}$ we investigate the electronic structure, dynamic mechanical stabilities and carrier mobilities of germanene dioxide bilayer as a noninteracting dielectric in van der Waals (vdW) electronics employing density functional theory (DFT) in this work. A number of low-energy structures had been investigated based on the of DFT optimization combined with the particle-swarm optimization (PSO) algorithm, and only the bilayer structure with space groups of $P 6 / \mathrm{mmm}$ of the most stable several 2D structures were obtained in our computations. ${ }^{33-36}$ The supercell in the $\alpha-2 \mathrm{D}$-germanene dioxide structure is shown in Fig. 1. The optimized lattice constant a is $4.00 \AA$ for $\alpha$-2D-germanene dioxide in a hexagonal unit cell, as enclosed in the gray dashed lines in Fig. 1a. $\alpha$-2D-germanene dioxide has an ideal $\mathrm{sp}^{3}$ bonding network, which means that 
(a)

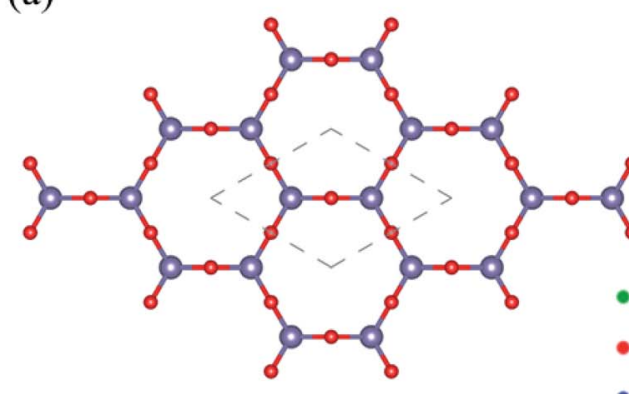

(b)

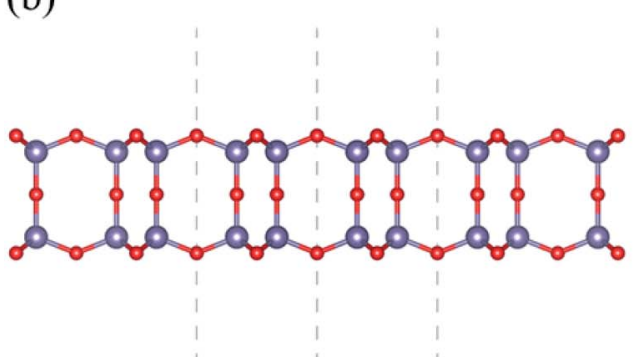

(c)

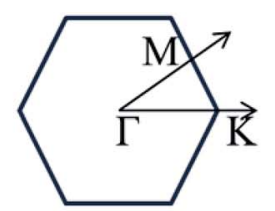

(d)
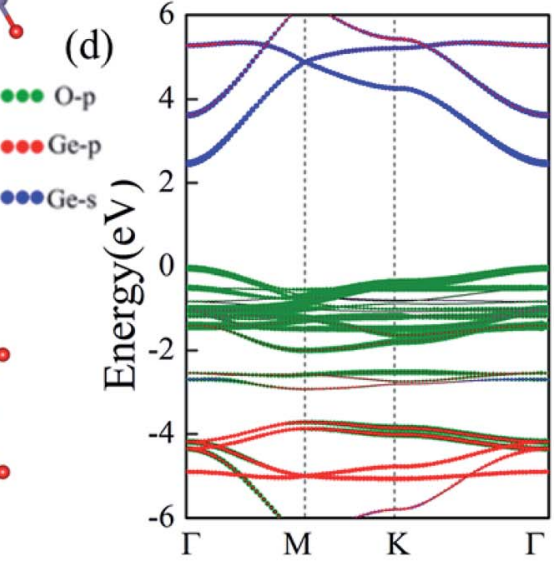

Fig. 1 Structure schematics of $\alpha-2 D$-germanene dioxide monolayer in a $2 \times 2$ supercell from the top (a) and side (b) views; the hexagonal primitive cells are enclosed in the gray dashed line. (d) Band structure of $\alpha$-2D-germanene dioxide monolayer computed at the PBE level. (c) Schematic diagram of the first Brillouin zone.

all $\mathrm{O}$ atoms are connected to Ge atoms with same solid angles $109^{\circ} 28^{\prime}$; i.e., $\mathrm{Ge}$ is in a perfect $\mathrm{O}$ tetrahedron. The new $\alpha-2 \mathrm{D}-$ germanene dioxide structure is dynamic and mechanical stabilities. Meanwhile, $\alpha$-2D-germanene dioxide has good stability, and can be stable up to $300 \mathrm{~K}$. These fantastic properties make $\alpha$-2D-germanene dioxide more attractive for electric and optical applications.

\section{Methods}

In the framework of DFT, ${ }^{37}$ the Vienna $\mathrm{Ab}$ initio Simulation Package (VASP) ${ }^{37,38}$ was used to optimize the initial structure using the method of projector augmented wave (PAW) ${ }^{39}$ In the general gradient approximation (GGA) around the optimization process, the exchange-correlation function for selecting Perdew-Burke-Ernzerhof (PBE) ${ }^{40,41}$ was used to relax the force of atoms in the system, until the force of each atom in the system was less than $0.1 \mathrm{eV} \mathrm{nm}^{-1}$. The cut-off kinetic energy is set to $360 \mathrm{eV}$. At the time of calculation, the doped systems were modeled with periodic geometry, growing along the $Y$-axis. Respectively, the Brillouin zone (BZ) was sampled using a $9 \times 9$ $\times 1$ gamma-centered Monkhorst-Pack grid, and the vacuum space was set to $30 \AA$ to minimize artificial interactions between neighboring slabs. ${ }^{42}$ All structures were fully optimized, including cell parameters and atomic coordinates. Phonon dispersion curves were obtained using the Phonopy package. ${ }^{43}$

\section{Results and discussion}

\section{Intrinsic structural and electronic properties}

We first present the geometric and electronic structures of the $\alpha$-2D-germanene dioxide, as shown in Fig. 1 . The $\alpha-2 D-$ germanene dioxide has a hexagonal honeycomb structure, and the relaxed lattice parameter is found to be $a=5.75 \AA$. The band structure is plotted in Fig. $1 \mathrm{~d}$. In the top view, $\alpha-2 \mathrm{D}$ germanene, like graphene, has a hexagonal crystal structure and an electronic performance like indirect-gap semiconductors. The $\alpha$-2D-germanene dioxide monolayer exhibits (quasi) direct-gap semiconducting character with a PBE- $E_{\mathrm{g}}$ of $2.50 \mathrm{eV}$ (see Fig. 1d). It has a larger band gap than $\mathrm{MoS}_{2}$ (bandgap $=1.9 \mathrm{eV}$ ), and is more easily used as short-wavelength light-emitting devices, ultraviolet detection and hightemperature, and high-power electronic devices. The valence band maximum (VBM) is based on the hybridized $\mathrm{O} 2 \mathrm{p}$ orbitals, while the conduction band minimum (CBM) derives from the hybridized Ge 4 s orbitals.

\section{Stability}

First, $\alpha$-2D-germanene dioxide was considered to determine its energetic stabilities. Fig. 2 shows the formation energy with area per Ge atom for germanene dioxide ( $\alpha$-2D-germanene dioxide). The formation energy of the $\alpha$-2D-germanene dioxide configuration with respect to its constituent atoms is calculated as:

$$
E_{\text {form }}=\left(E_{\text {total }}-N_{\mathrm{Ge}} E_{\mathrm{Ge}}-N_{\mathrm{O}} E_{\mathrm{O}}\right) /\left(N_{\mathrm{Ge}}+N_{\mathrm{O}}\right)
$$

where $E_{\text {total }}$ is the total energy of the system. $E_{\mathrm{Ge}}$ and $E_{\mathrm{O}}$ are the energies of single $\mathrm{Ge}$ and $\mathrm{O}$ in bulk $\mathrm{Ge}$ and $\mathrm{O}_{2}$ molecules, which are calculated to be -0.765 and $-1.842 \mathrm{eV}$, respectively. $N_{\mathrm{Si}}$ and $N_{\mathrm{O}}$ are the numbers of $\mathrm{Si}$ and $\mathrm{O}$ atoms in the unit cell. A negative formation energy suggests that the configuration is stable. ${ }^{44}$ Note that the energy minimum in each curve of Fig. 2c represents the equilibrium configuration of the given structure. The calculated formation energies of $\alpha$-2D-germanene dioxide are 
(a)

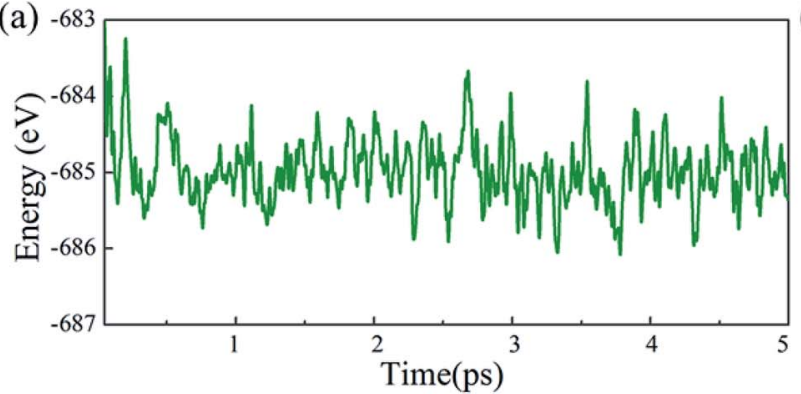

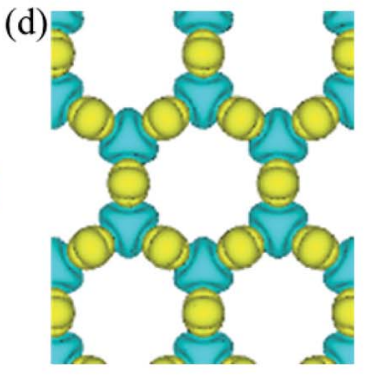

(b)

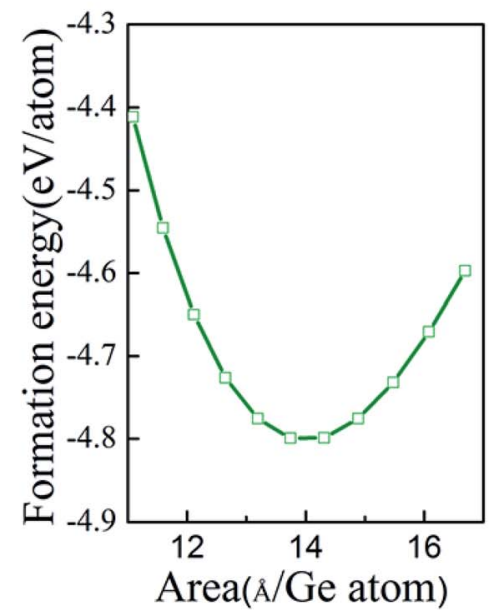

(c)

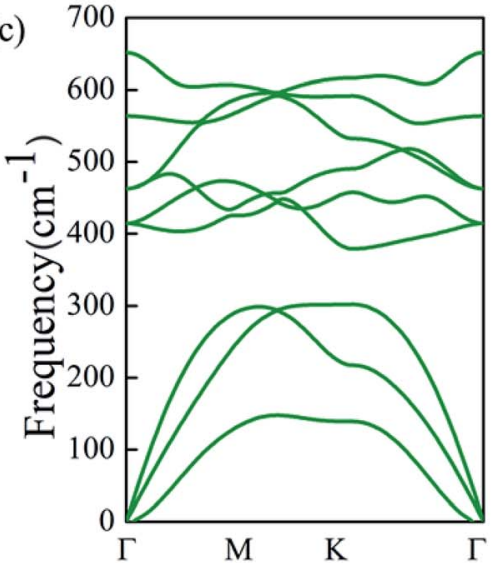

Fig. 2 (a) Variations of the free energy from 1 to 5 ps during AIMD at the temperature of $300 \mathrm{~K}$. (b) Calculated formation energy vs. area/Ge atom for $\alpha$-2D-germanene dioxide. (c) Phonon dispersion curves. (d) Deformation charge density (with isosurface of 0.01 e $\AA^{-3}$ ).

$-4.80 \mathrm{eV}$ per atom (Fig. 2b). These results suggest that the $\alpha-2 \mathrm{D}-$ germanene configuration is either stable or metastable.

Next, the thermal stabilities of the germanene dioxide configurations are calculated by $a b$ initio molecular dynamics (AIMD) simulations in Fig. 2a. The MD simulations selected the supercell of $4 \times 4 \times 1$ based on the Nose thermostat at $500 \mathrm{~K}^{45}$ The phonon dispersion (based on Vibra of SIESTA utility ${ }^{\mathbf{4 6}}$ ) curves show the dynamic stability of $\alpha$-2D-germanene dioxide (Fig. 2c). The deformation charge density shown in Fig. 2d suggests the charge transfer from $\mathrm{Ge}$ to O yielding the charge of Si to be 3.52e by Bader analysis. The same structure of the silica sheet in Russia (0001) (i.e. double silicon layer) was recently synthesized. ${ }^{\mathbf{1 4}}$ This indicates that our material is likely to be prepared.

\section{Carrier mobility}

The carrier mobility $(\mu)$ of $\alpha$-2D-germanene dioxide is calculated based on the deformation potential (DP) ${ }^{47}$ theory as proposed by Bardeen and Shockley, which has been proven to be reasonable in estimating $\mu$ of $2 \mathrm{D}$ materials. Thus, the carrier mobility $(\mu)$ of $\alpha$-2D-germanene dioxide, as predicted using the DP theory, is calculated as:

$$
\mu_{2 \mathrm{D}}=\frac{2 e \hbar^{3} C_{2 \mathrm{D}}}{3 k_{\mathrm{B}} T\left|m^{*}\right|^{2} E_{1}{ }^{2}}
$$

where $e$ is the electron charge, $\hbar$ is reduced Planck, $k_{\mathrm{B}}$ is Boltzmann's constant and $T$ is the temperature which set to $300 \mathrm{~K}$, while $m^{*}, E_{1}$, and $C_{2 \mathrm{D}}$ are the effective mass, deformation potential constant, and in-plane stiffness, which will appear in the next calculation. The effective masses $\left(m^{*}=\hbar^{2}\left(\partial^{2} E / \partial K^{2}\right)^{-1}\right)$ of electrons and holes associated with the direct semiconducting $\alpha-2 \mathrm{D}$ germanene dioxide are computed. Interestingly, the effective mass of electrons in the $k_{\mathrm{a}}$ direction $\left(0.05 \mathrm{~m}_{\mathrm{e}}\right)$ is much smaller than that in the $k_{\mathrm{b}}$ direction $\left(0.07 \mathrm{~m}_{\mathrm{e}}\right)$, indicating the easy drift of electrons in the $k_{\mathrm{a}}$ direction. However, the holes entail an effective mass of $\sim 0.41 / 0.053 m_{\mathrm{e}}\left(k_{\mathrm{a}} / k_{\mathrm{b}}\right)$, suggesting the easy drift of holes in the $k_{\mathrm{b}}$ direction. In addition, the small effective mass in a direction could promise high carrier mobility for $\alpha$-2D-germanene dioxide in this direction. By fitting the change of total energy $(E)$ versus the uniaxial strain $(\varepsilon)$, as shown in Fig. 3a, the in-plane stiffness $C_{2 \mathrm{D}}=$ $\left[\partial^{2} E / \partial \delta^{2}\right] / S_{0} . S_{0}$, the area of $\alpha$-2D-germanene dioxide along $a$ and $b$ directions, are evaluated to be 96.73 and $99.21 \mathrm{~N} \mathrm{~m}^{-1}$, respectively, as shown in Table 1 . The absolute DP constant $E_{1}\left(=\mathrm{d} E_{\text {edge }} /\right.$ $\mathrm{d} \delta$ ) for electrons along $a$ and $b$ directions is calculated to be 16.11 and $14.53 \mathrm{eV}$, respectively, as shown in Table 1 . According to the calculated effective mass $m^{*}, C_{2 \mathrm{D}}$ and $E_{1}$, the electron mobility can be evaluated based on Table 1 . Interestingly, the holes mobility in the $k_{\mathrm{a}}$ direction can be as high as $4.09 \times 10^{3} \mathrm{~cm}^{2} \mathrm{~V}^{-1} \mathrm{~s}^{-1}$, which is much higher than that of $\operatorname{MoS}_{2}\left(\sim 3.0 \mathrm{~cm}^{2} \mathrm{~V}^{-1} \mathrm{~s}^{-1}\right)^{48}$ at room temperature. Therefore, the high carrier mobility found in the study is of great significance to the study of electron transport.

\section{Optical properties}

The optical properties are tied closely to the dielectric function which can be calculated by: 

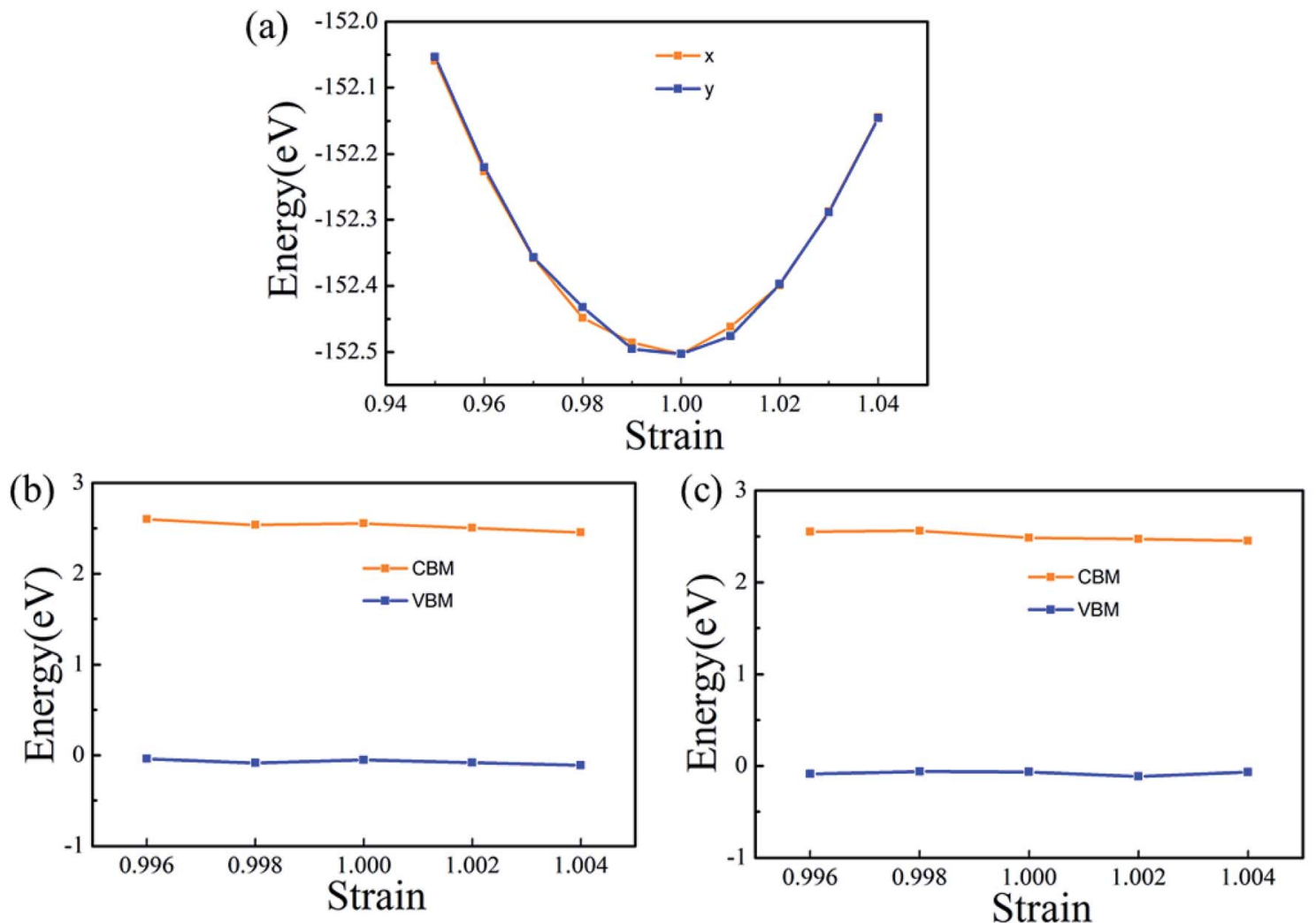

Fig. 3 (a) Energy difference between the total energy of the monolayer under uniaxial strain and that of monolayer under no strain as a function of strain along $k_{\mathrm{a}}$ and $k_{\mathrm{b}}$ directions. The in-plane stiffness $C_{2 \mathrm{D}}$ can be obtained after fitting the parabola. The energy of VBM and CBM shift with respect to the lattice dilation and compression along $x(b)$ and $y(c)$ directions. The slopes of the straight lines correspond to the DP constant along different directions for electrons and holes.

Table 1 Calculated effective mass $|m *|$, DP constant $E_{1}$, in-plane stiffness $C_{2 \mathrm{D}}$, carrier mobility $\mu$, and relaxation time $\tau$ of GaGeTe monolayer along the $A C$ and $Z Z$ directions

\begin{tabular}{llllll}
\hline Direction & Carrier type & $\left|m^{*}\right|\left(m_{\mathrm{e}}\right)$ & $\left|E_{1}\right|(\mathrm{eV})$ & $C_{2 \mathrm{D}}\left(\mathrm{N} \mathrm{m}^{-1}\right)$ & $\mu\left(\mathrm{cm}^{2}\left(\mathrm{~V}^{-1} \mathrm{~s}^{-1}\right)\right)$ \\
\hline$k_{\mathrm{a}}$ & Holes & 0.41 & 11.08 & 96.73 & $4.09 \times 10^{3}$ \\
& Electrons & 0.05 & 16.11 & & $2.12 \times 10^{3}$ \\
$k_{\mathrm{b}}$ & Holes & 0.05 & 11.56 & 99.21 & $3.76 \times 10^{3}$ \\
& Electrons & 0.07 & 14.53 & & $1.36 \times 10^{3}$
\end{tabular}

$$
\varepsilon(\omega)=\varepsilon_{1}(\omega)+\mathrm{i} \varepsilon_{2}(\omega)
$$

where the $\varepsilon_{1}(\omega)$ and $\varepsilon_{2}(\omega)$ are the real and imaginary part of the function, and $\varepsilon_{1}(\omega)$ can be derived from the imaginary part, $\varepsilon_{2}(\omega)$, the dielectric function by Kramer-Kronig, can be expressed as: ${ }^{\mathbf{2}}$

$\varepsilon_{2}(\omega)=\frac{4 \pi^{2}}{m^{2} \omega^{2}} \sum_{\mathrm{V}, \mathrm{C}} \int_{\mathrm{BZ}} d^{3} k \frac{2}{2 \pi}\left|e M_{\mathrm{CV}}(K)\right|^{2} \times \delta\left[E_{\mathrm{C}}(k)-E_{\mathrm{V}}(k)-\hbar \omega\right]$

In addition, the absorption coefficient $I(\omega)$ was obtained by:

$$
I(\omega)=\sqrt{2} \omega\left[\sqrt{\varepsilon_{1}^{2}(\omega)-\varepsilon_{2}^{2}(\omega)}-\varepsilon_{1}(\omega)\right]^{1 / 2}
$$

where the $\mathrm{C}$ is the conduction band, $\mathrm{V}$ is valence band states, $\Omega$ is the unit-cell volume, $m$ is the mass of free electrons, $e$ is the charge of free electrons, and $\omega$ is the frequency of incident photons.

The properties of materials are also directly reflected in the optical properties. The wide bandgap of $\alpha$-2D-germanene dioxide means that it can more easily be used in shortwavelength light-emitting devices and ultraviolet detection compared with $\mathrm{MoS}_{2}$. Attracted by the proper band gap and excellent electronic properties of $\alpha$-2D-germanene dioxide, we further explored the light absorption of $\alpha$-2D-germanene dioxide. As shown in Fig. $4 b, \alpha$-2D-germanene dioxide shows absorption starting at $\sim 2.5 \mathrm{eV}$, with two main absorption peaks from $\sim 5.5$ to $\sim 7.5 \mathrm{eV}$, corresponding to significant light absorption at the ultraviolet region of the solar spectrum. The 

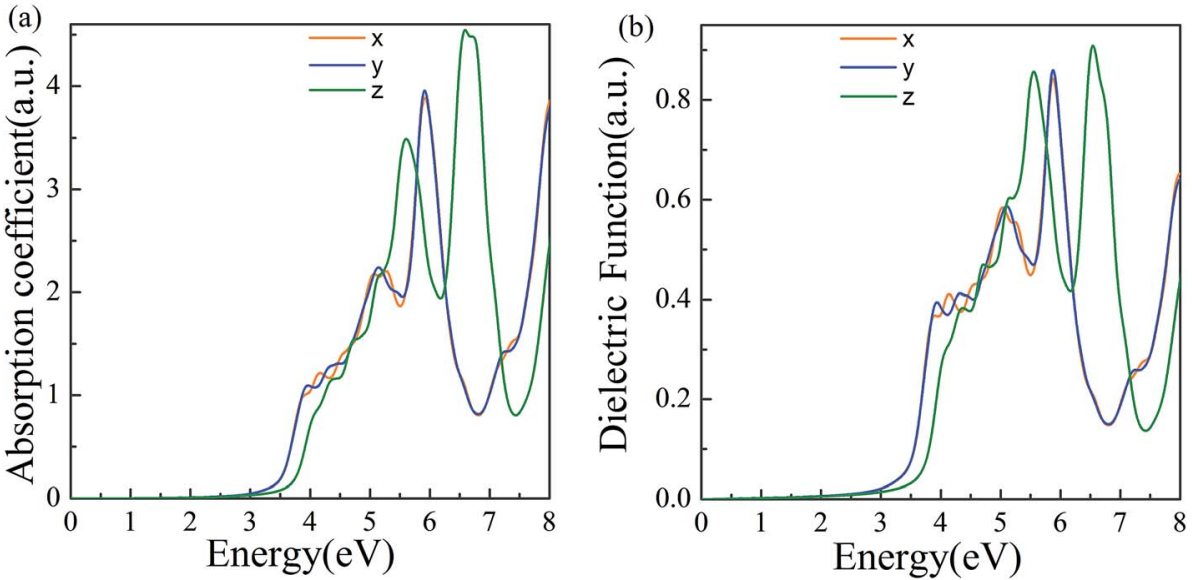

Fig. 4 (a) Computed imaginary absorption coefficient for $\alpha$-2D-germanene dioxide along different incident light directions. (b) Computed imaginary dielectric functions versus energy.

incident light can be effectively absorbed in three directions, with the adsorption coefficients increasing up to the order of 4.5 a.u., as shown in Fig. 4a. As shown in Fig. 4b, the plot of dielectric functions versus energy shows the same trend. Therefore, $\alpha$-2D-germanene dioxide seems rather attractive for efficient light harvesting and may have promising applications in optoelectronics.

\section{Conclusions}

In conclusion, based on first-principles calculations, we show that $\alpha$-2D-germanene dioxide has an appropriate band gap and excellent electronic and optical properties, attracting great attention as a possible candidate for electronic devices. $\alpha-2 \mathrm{D}-$ germanene dioxide shows semiconductor properties, with a gap reaching up to $2.50 \mathrm{eV}$. To our delight, $\alpha$-2D-germanene dioxide is fairly stable according to calculations of formation energy, MD simulation and phonon dispersion curves. $\alpha-2 D-$ germanene dioxide possesses a very high electron mobility of $4.09 \times 10^{3} \mathrm{~cm}^{2} \mathrm{~V}^{-1} \mathrm{~s}^{-1}$ along the $k_{\mathrm{a}}$ direction, which is much higher than that of $\operatorname{MoS}_{2}\left(\sim 3.0 \mathrm{~cm}^{2} \mathrm{~V}^{-1} \mathrm{~s}^{-1}\right)$. Furthermore, the excellent optical properties makes $\alpha$-2D-germanene dioxide an excellent optical device. Prominent stability, appropriate band gap, high carrier mobility and remarkable light absorption render the studied materials highly suitable for applications in electronics and optoelectronics.

\section{Conflicts of interest}

There are no conflicts to declare.

\section{Acknowledgements}

This work was supported by the National Natural Science Foundation of China (Grant Nos. 61571210, 61172028, and 11434006), and Shandong Provincial Natural Science Foundation of China (Grant No. ZR2018QA006).

\section{References}

$1 \mathrm{~S}$. Shaikhutdinov and H.-J. Freund, Ultrathin silica films on metals: the long and winding road to understanding the atomic structure, Adv. Mater., 2013, 25, 49-67.

2 T. Demuth, Y. Jeanvoine, J. Hafner and J. Angyan, Polymorphism in silica studied in the local density and generalized-gradient approximations, J. Phys.: Condens. Matter, 1999, 11, 3833.

3 X. Q. Chen, H. Y. Niu, D. Li and Y. Li, Modeling hardness of polycrystalline materials and bulk metallic glasses, Intermetallics, 2011, 9, 1275-1281.

4 S. Stishov and N. Belov, Crystal structure of a new dense modification of silica $\mathrm{SiO}_{2}$, Dokl. Akad. Nauk SSSR, 1962, 143, 951.

5 E. Chao, J. Fahey, J. Littler and D. S. Milton, $\mathrm{SiO}_{2}$, a very high pressure new mineral from Meteor Crater, Arizona, $J$. Geophys. Res., 1962, 67, 419-421.

6 D. M. Teter, R. J. Hemley, G. Kresse and J. Hafner, High pressure polymorphism in silica, Phys. Rev. Lett., 1998, 80, 2145.

7 S. R. Shieh, T. S. Duffy and B. Li, Strength and elasticity of $\mathrm{SiO}_{2}$ across the stishovite- $\mathrm{CaCl}_{2}$-type structural phase boundary, Phys. Rev. Lett., 2002, 89, 255507.

8 G. X. Wang, G. C. Loh, R. Pander and S. P. Karna, Novel TwoDimensional Silica Monolayers with Tetrahedral and Octahedral Configurations, J. Phys. Chem. C, 2015, 119, 15654-15660.

9 V. Prakapenka, G. Shen, L. Dubrovinsky, M. Rivers and S. Sutton, High pressure induced phase transformation of $\mathrm{SiO}_{2}$ and $\mathrm{GeO}_{2}$ : difference and similarity, J. Phys. Chem. Solids, 2004, 65, 1537-1545.

10 T. K. Todorova, M. Sierka, J. Sauer, S. Kaya, J. Weissenrieder, J. Lu, H. J. Gao, S. Shaikhutdinov and H. J. Freund, Erratum: atomic structure of a thin silica film on a Mo(112) substrate: a combined experimental and theoretical study, Phys. Rev. B: Condens. Matter Mater. Phys., 2006, 73, 165414. 
11 J. Weissenrieder, S. Kaya, J.-L. Lu, H.-J. Gao, S. Shaikhutdinov, H.-J. Freund, M. Sierka, T. K. Todorova and J. Sauer, Atomic structure of a thin silica film on a $\mathrm{Mo}(112)$ substrate: a two-dimensional network of $\mathrm{SiO}_{4}$ tetrahedra, Phys. Rev. Lett., 2005, 95, 076103.

12 T. Schroeder, M. Adelt, B. Richter, M. Naschitzki, M. Bäumer and H.-J. Freund, Growth of well-ordered silicon dioxide films on Mo(1 1 2), Surf. Rev. Lett., 2000, 7, 7-14.

13 J. Seifert, D. Blauth and H. Winter, Evidence for 2D-Network Structure of Monolayer Silica Film on Mo(112), Phys. Rev. Lett., 2009, 103, 017601.

14 D. Löffler, J. J. Uhlrich, M. Baron, B. Yang, X. Yu, L. Lichtenstein, L. Heinke, C. Büchner, M. Heyde, S. Shaikhutdinov, H.-J. Freund, R. Włodarczyk, M. Sierka and J. Sauer, Growth and structure of crystalline silica sheet on $\mathrm{Ru}(0001)$, Phys. Rev. Lett., 2010, 105, 146104.

15 M. Heyde, S. Shaikhutdinov and H.-J. Freund, Twodimensional silica: crystalline and vitreous, Chem. Phys. Lett., 2012, 550, 1-7.

16 P. Y. Huang, S. Kurasch, A. Srivastava, V. Skakalova, J. Kotakoski, A. V. Krashenin-nikov, R. Hovden, Q. Mao, J. C. Meyer, J. Smet, D. A. Muller and U. Kaiser, Direct imaging of a two-dimensional silica glass on grapheme, Nano Lett., 2012, 12, 1081-1086.

17 A. K. Geim, I. V. Grigorieva, A. K. Geim and I. V. Grigorieva, Nature, 2013, 499, 419-425.

18 P. Miro, M. Audiffred and T. Heine, An atlas of twodimensional materials, Chem. Soc. Rev., 2014, 43, 6537-6554.

19 K. Zheng, X. Yang, H. Cui, Q. Yang, H. Ye, D. Xiong, $\mathrm{S}$. Ingebrandt and $\mathrm{X}$. Chen, Intriguing Electronic Insensitivity and High Carrier Mobility in Monolayer Hexagonal, J. Mater. Chem. C, 2018, 6, 4943.

20 S. Guo, Z. Zhu, X. Hu, W. Zhou, X. Song, S. Zhang, K. Zhang and H. Zeng, Tailored design of functional nanoporous carbon materials toward fuel cell applications, Nanoscale, 2018, 10, 8397.

21 Z. Gao, X. Dong, N. Li and J. Ren, Controllable Electrochemical Synthesis of Hierarchical $\mathrm{ZnO}$ Nanostructures on FTO Glass, Nano Lett., 2017, 17, 772.

22 J. Jiang, Q. Liang, R. Meng, Q. Yang, C. Tan, X. Sun and $\mathrm{X}$. Chen, Exploration of new ferromagnetic, semiconducting and biocompatible $\mathrm{Nb}_{3} \mathrm{X}_{8}(\mathrm{X}=\mathrm{Cl}, \mathrm{Br}$ or I) monolayers with considerable visible and infrared light absorption, Nanoscale, 2017, 9, 2992.

23 P. Vogt, P. D. Padova, C. Quaresima, J. Avila, E. Frantzeskakis, M. C. Asensio, A. Resta, B. Ealet and G. L. Lay, Silicene: compelling experimental evidence for graphene like two-dimensional silicon, Phys. Rev. Lett., 2012, 108, 155501.

24 A. K. Geim and K. S. Novoselov, Giant nonlocality near the dirac point in graphene, Nat. Mater., 2007, 6, 183.

25 J. Zhang, L. Wang, Q. Liu, H. Li, D. Yu, W. Mei, J. Shi, Z. Gao and J. Lu, Sub-10 nm Gate Length Graphene Transistors: Operating at Terahertz Frequencies with Current Saturation, Sci. Rep., 2013, 3, 1314.
26 C. W. Zhang and S. S. Yan, First-principles Study of Ferromagnetism in Two-Dimensional Silicene with Hydrogenation, J. Phys. Chem. C, 2012, 116, 4163.

27 R. W. Zhang, C. W. Zhang, S. S. Li, W. X. Ji and P. J. Wang, Tunable electronic properties in the van der waals heterostructure of germanene/germanene, Phys. Chem. Chem. Phys., 2015, 17, 12194-12198.

28 R. W. Zhang, C. W. Zhang, W. X. Ji and S. S. Yan, Silicane as an Inert Substrate of Silicene: A Promising Candidate for FET, J. Phys. Chem. C, 2014, 118, 25278-25283.

29 Z. Gao, X. Dong, N. Li and J. Ren, Novel Two-Dimensional Silicon Dioxide with in-plane Negative Poisson's Ratio, Nano Lett., 2017, 17, 772-777.

30 P. Vogt, P. P. De, C. Quaresima, J. Avila, E. Frantzeskakis, M. C. Asensio, A. Resta, B. Ealet and G. Le Lay, Silicene: Compelling experimental evidence for graphene like twodimensional silicon, Phys. Rev. Lett., 2012, 108, 489-492.

31 L. C. L. Y. Voon, E. Sandberg, R. S. Aga and A. A. Farajian, Hydrogen compounds of group-IV nanosheets, Appl. Phys. Lett., 2010, 97, 163114.

32 Y. M. Dou, W. X. Ji, S. F. Zhang, C. W. Zhang, P. Li and P. J. Wang, Novel 2D Germanene Dioxide Monolayers: Mechanical Properties, Hole-Mobility Values, and Carrier Mobility, Ann. Phys., 2018, 9, 1800214.

33 Y. Wang, J. Lv, L. Zhu and Y. Ma, Crystal structure prediction via particle-swarm optimization, Phys. Rev. B: Condens. Matter Mater. Phys., 2010, 82, 094116.

34 Y. Wang, J. Lv, L. Zhu and Y. Ma, CALYPSO: a method for crystal structure prediction, Comput. Phys. Commun., 2012, 183, 2063-2070.

35 G. Kresse and J. Furthmüller, Efficiency of ab-initio total energy calculations for metals and semiconductors using a plane-wave basis set, Comput. Mater. Sci., 1996, 6, 15-50.

36 G. Kresse and D. Joubert, From ultrasoft pseudopotentials to the projector augmented-wave method, Phys. Rev. B: Condens. Matter Mater. Phys., 1999, 59, 1758-1775.

37 M. Houssa, B. Van den Broek, E. Scalise, G. Pourtois, V. V. Afanas'ev and A. Stesmans, An electric field tunable energy band gap at silicene/(0001) ZnS interfaces, Phys. Chem. Chem. Phys., 2013, 15, 3702-3705.

38 G. Kresse and J. Furthmuller, Efficient iterative schemes for ab initio total-energy calculations using a plane-wave basis set, Phys. Rev. B: Condens. Matter Mater. Phys., 1996, 54, 11169-11186.

39 G. Kresse and D. Joubert, From Ultrasoft Pseudopotential to the Projector Augmented-Wave Method, Phys. Rev. B: Condens. Matter Mater. Phys., 1999, 59, 1758-1775.

40 J. P. Perdew, A. Ruzsinszky, G. I. Csonka, O. A. Vydrov and G. E. Scuseria, Erratum: Restoring the Density-Gradient Expansion for Exchange in Solids and Surfaces, Phys. Rev. Lett., 2008, 100, 136406.

41 J. P. Perdew, K. Burke and M. Ernzerhof, Erratum: Generalized Gradient Approximation Made Simple, Phys. Rev. Lett., 1996, 77, 3865.

42 H. J. Monkhorst and J. D. Pack, Special points for Brillouinzone integrations, Phys. Rev. B: Solid State, 1976, 13, 51885192. 
43 A. Togo, F. Oba and I. Tanaka, First-principles calculations of the ferroelastic transition between rutile-type and $\mathrm{CaCl}_{2}$ type $\mathrm{SiO}_{2}$ at high pressures, Phys. Rev. B: Condens. Matter Mater. Phys., 2008, 78, 134106.

44 Q. Li, D. Zhou, W. Zheng, Y. Ma and C. Chen, Global structural optimization of tungsten borides, Phys. Rev. Lett., 2013, 110, 136403.

45 S. Nosé, A unified formulation of the constant temperature molecular dynamics methods, J. Chem. Phys., 1984, 81, 511.
46 A. Postnikov, O. Pagès and J. Hugel, Lattice dynamics of the mixed semiconductors (Be, Zn) Se from first-principles calculations, Phys. Rev. B: Condens. Matter Mater. Phys., 2005, 71, 115206.

47 A. Togo, F. Oba and I. Tanaka, First-principles calculations of the ferroelastic transition between rutile-type and $\mathrm{CaCl}_{2}$ type $\mathrm{SiO}_{2}$ at high pressures, Phys. Rev. B: Condens. Matter Mater. Phys., 2008, 78, 134106.

48 H. Zhang, Y. Ma and Z. Chen, Quantum spin hall insulators in strain-modified arsenene, Nanoscale, 2015, 7, 19152. 